\title{
Renal artery stenosis in a young female patient with severe hypertension - a case report
}

Giuliano De Stefano, Federica De Pisapia, Giovanni Albano, Maria Immacolata Arnone, Giovanni Esposito, Giovanni de Simone, Nicola De Luca, Costantino Mancusi

Hypertension Research Center, Federico II University Hospital, Napoli, Italy

\begin{abstract}
Renal artery stenosis is a frequent cause of secondary hypertension, but the diagnostic and therapeutic management of these hypertensive patients is controversial. We report a case of secondary hypertension due to renal artery stenosis, treated with the implantation of a drug-eluting stent.
\end{abstract}

\section{Introduction}

About $5-15 \%$ of patients with hypertension have a secondary cause, which may be treatable with a specific intervention. ${ }^{1}$

Renal artery stenosis (RAS) is one of the most important causes of secondary hypertension, and affects 1 to $5 \%$ of all hypertensive patients. ${ }^{2}$ Atherosclerosis and fibromuscular dysplasia are the most common etiologies; less frequent causes of RAS are Takayasu arteritis, renal artery entrapment by the diaphragmatic crus, type 1 neurofibromatosis, vascular Ehlers-Danlos syndrome and other congenital diseases; ${ }^{3}$ strategies of treatment include medical therapy, percutaneous interventions with balloon angioplasty or stent implantation and surgery, but the identification of the best approach remains controversial.

Correspondence: Costantino Mancusi, Hypertension Research Center, Federico II University Hospital, via Pansini 5, 80131 Napoli, Italy.

E-mail: costantino.mancusi@unina.it

Key words: Blood pressure; renal artery stenosis; hypertension; secondary hypertension; ambulatory blood pressure monitoring; drug-eluting stent; renin; renal doppler ultrasound.

Conflict of interests: the authors declare no potential conflict of interests.

Received for publication: 4 February 2019.

Revision received: 9 May 2019.

Accepted for publication: 3 June 2019.

This work is licensed under a Creative Commons Attribution NonCommercial 4.0 License (CC BY-NC 4.0).

${ }^{\circ}$ Copyright: the Author(s), 2019

Licensee PAGEPress, Italy

Italian Journal of Medicine 2019; 13:176-180

doi:10.4081/itjm.2019.1143

\section{Case Report}

A 27-year-old woman was referred to our outpatient clinic to perform a screening for secondary hypertension, after incidental detection of elevated blood pressure during hospitalization for cholecystectomy.

On admission, blood pressure was 160/110 $\mathrm{mmHg}$, without relevant information from clinical history (absence of: sweating, pounding headache, palpitations or flushing; variation of body weight; smoking status or use of medications and illicit drugs; family history of secondary hypertension) and electrocardiogram. Physical examination did not provide important findings: her weight was normal, and she did not show any abdominal bruit; her blood pressure did not differ between upper-lower extremities or between right-left arm. She underwent $24 \mathrm{~h}$ ambulatory blood pressure monitoring (ABPM), which showed mean awake values of $147 / 111 \mathrm{mmHg}$, a peak pressure of $176 / 133 \mathrm{mmHg}$ late in the evening and dipping pattern, with mean asleep values of $131 / 91 \mathrm{mmHg}$ (Figure 1, panel A).

She underwent standard Doppler echocardiography, ${ }^{4}$ which showed no evidence of cardiac target organ damage.

A full screening for secondary causes of elevated blood pressure was scheduled, including laboratory tests (complete blood count, potassium, sodium, creatinine, fasting glucose, fasting lipid profile, urinalysis, thyroid-stimulating hormone, renin and aldosterone plasma levels, $24 \mathrm{~h}$ urinary free cortisol, erythrocyte sedimentation rate, and C-reactive protein), carotid and peripheral vascular ultrasonography, renal ultrasound for evaluation of renal arterial blood flow. Antihypertensive treatment with amlodipine and then doxazosin was given. Elevated levels of plasma renin and aldosterone were found (normal aldosterone-to-renin ratio) with normal renal function and electrolytes (Table 1). Carotid and peripheral vascular 
ultrasound did not show any alteration. Renal ultrasound and doppler ultrasound of renal arteries showed reduced size of right kidney, with normal parenchymal morphology; normal size and structure of left kidney; no evidence of hydronephrosis or calculus. Left renal artery showed normal peak systolic velocity and normal flow of intrarenal vasculature. Right renal artery was not well visualized at the origin, but demonstrated a tardus-parvus pattern waveform at the interlobar arteries. On suspicion of renal artery steno- sis due to fibromuscular dysplasia, a magnetic resonance angiogram was done and revealed right renal artery stenosis, confirmed by subsequent invasive angiography, right before the bifurcation with a polar superior branch, with mild post stenotic dilatations (Figure 2, panel A).

We made the decision to implant a drug-eluting stent due to close anatomical proximity of the diaphragmatic pillar, with optimal resolution of the stenosis (Figure 2, panel B). Dual antiplatelet therapy
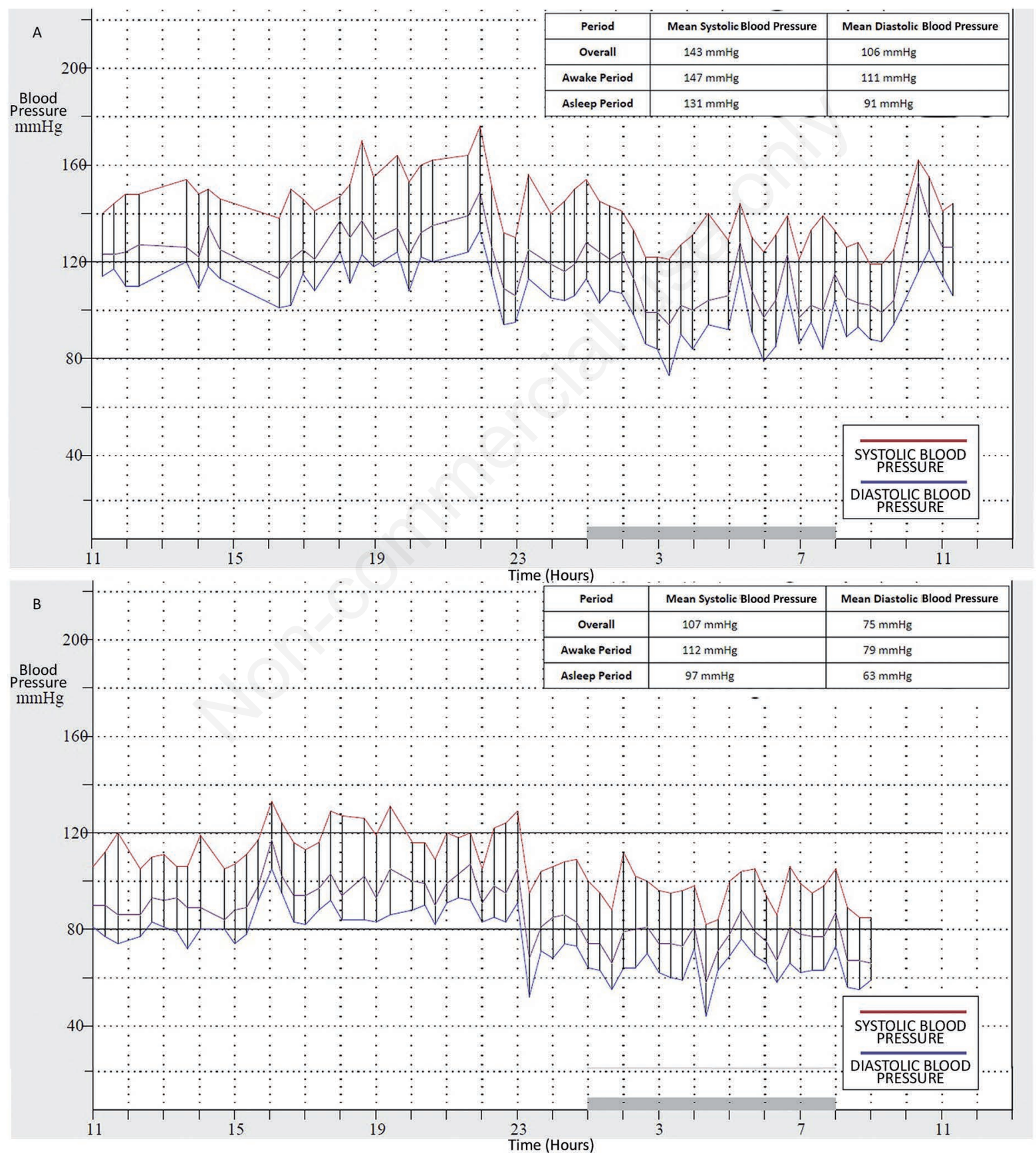

Figure 1. Ambulatory blood pressure monitoring result A) before and B) after stenting. 
was started. The first day after stenting, the patient's blood pressure was $110 / 70 \mathrm{mmHg}$ without medical therapy, her renal function was unchanged, her renin plasma concentration and Doppler intrarenal blood flow were normalized (Table 1). After 40 days of follow-up, the patient was asymptomatic, referred normal blood pressure at home monitoring without any antihypertensive drug; her renal function and renin/aldosterone plasmatic levels persisted in normal range. We performed ABPM that showed excellent blood pressure control during $24 \mathrm{~h}$ (Figure 1, panel B). During the last follow-up, after 10 months, the patient exhibited a home blood pressure monitoring of 120/75 $\mathrm{mmHg}$, normal renal function and serum electrolytes. We performed a renal ultrasound with evidence of a normal pattern at Doppler evaluation of right intrarenal vasculature, and an ABPM that confirmed the optimal blood pressure control with mean awake values of $113 / 80 \mathrm{mmHg}$, mean asleep values of $96 / 63 \mathrm{mmHg}$ and overall values of 107/74 mmHg (Figure 3). Dual antiplatelet therapy was stopped after 12 months, without any adverse event.

\section{Discussion}

Secondary hypertension, defined as hypertension due to an identifiable cause, is commonly related to conditions as renovascular disease, obstructive sleep apnea, endocrine causes (primary aldosteronism, pheochromocytoma, Cushing's syndrome, thyroid disease), coarctation of the aorta, genetic causes, medications or illicit drugs and affects about $5-15 \%$ of all subjects with hypertension.

Screening all hypertensive patients is not feasible; however, in the presence of suggestive characteristics (age $<40$ years, resistant or severe hypertension, acute worsening of hypertension in a patient with previously stable control, hypertension emergency, extensive hypertension-mediated organ damage, clinical features suggestive of obstructive sleep apnea, family history of pheochromocytoma) screening should be considered after confirming that blood pressure is elevated with ABPM. ${ }^{1}$

Through screening we excluded primary aldosteronism because of normal aldosterone-to-renin ratio sup-

Table 1. Laboratory tests.

\begin{tabular}{|c|c|c|c|c|}
\hline & Pre-stenting & Post-stenting & Follow-up (40 days) & Reference range \\
\hline Supine plasma renin $(\mathrm{pg} / \mathrm{mL})$ & 450 & 23.4 & 15.8 & $2.52-35.82$ \\
\hline Upright plasma renin (pg/mL) & 582 & 45.8 & 28.1 & $3.1-59.5$ \\
\hline Supine plasma aldosterone $(\mathrm{pg} / \mathrm{mL})$ & 808 & $<37$ & 69 & $40-310$ \\
\hline Upright plasma aldosterone (pg/mL) & 758 & 187 & 125 & $40-432$ \\
\hline Serum creatinine $(\mathrm{mg} / \mathrm{dL})$ & 0.7 & 0.7 & 1 & $0.6-1.1$ \\
\hline Serum sodium $(\mathrm{mmol} / \mathrm{L})$ & 139 & 139 & 138 & $136-145$ \\
\hline Serum potassium $(\mathrm{mmol} / \mathrm{L})$ & 3.8 & 4.1 & 3.9 & $3.5-5.1$ \\
\hline
\end{tabular}
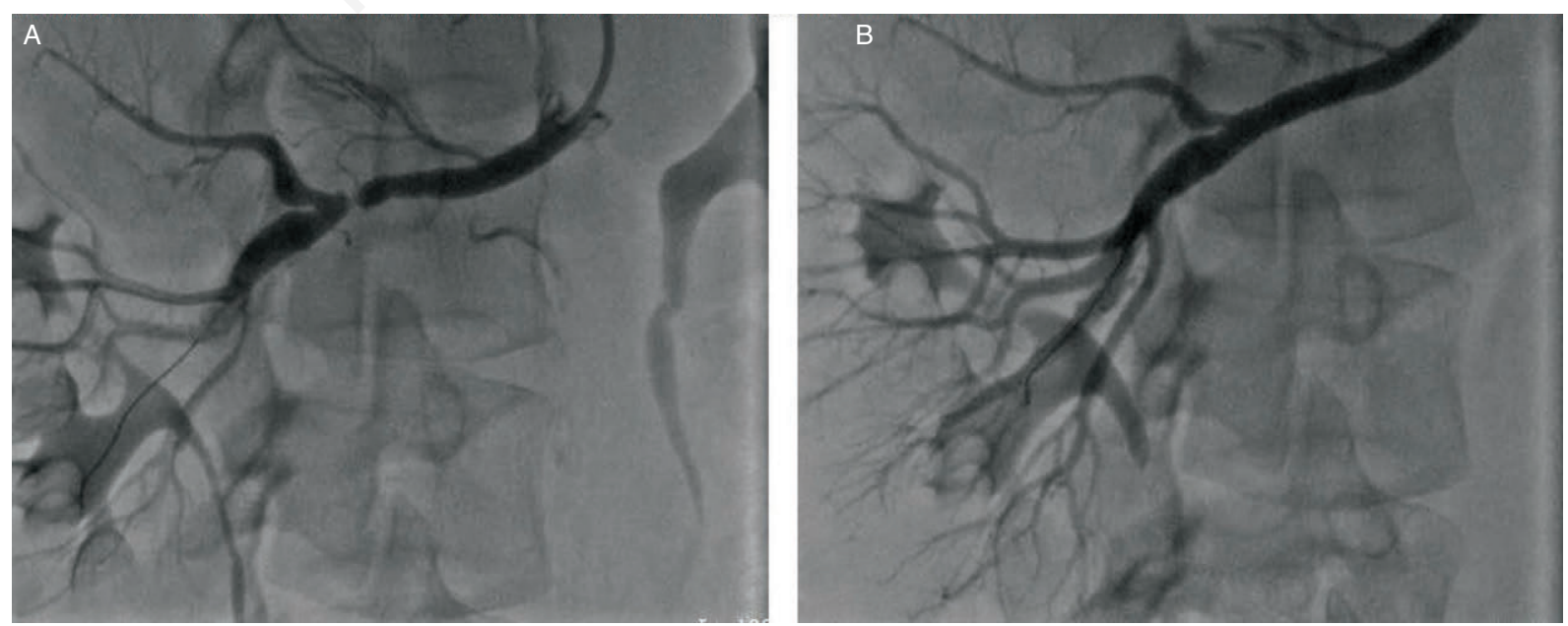

Figure 2. Renal arteriogram $A$ ) before and B) after stenting. 
ported by normokalemia, although only a minority of patients (about 30\%) have hypokalemia. ${ }^{5}$ Other endocrine causes of secondary hypertension have been excluded given the normal function test, while coarctation of the aorta have been excluded using echocardiography. Further investigations revealed right renal artery stenosis, and the absence of atherosclerotic plaque or vascular thickening, diabetes or dyslipidemia, the normality of measures of systemic inflammation, the lack of familial or syndromic disease, and the young age established as most likely diagnosis renal artery stenosis from unifocal fibromuscular dysplasia. ${ }^{3}$

RAS is a frequent cause of secondary hypertension, and affects 1 to $5 \%$ of all hypertensive patients. Regardless of etiology the management of patients with RAS is not well defined and a real challenge for clinicians is to identify patients with renovascular disease who can potentially benefit from revascularization.

ABPM is a better predictor of cardiovascular mortality than clinic measurement ${ }^{6}$ and is useful to assess the severity of hypertension and the response to treatment.?

Data in the current literature concerning the role of ABPM before and after RAS revascularization are not frequent, but the available evidence demonstrates that a finding of high blood pressure values at ABPM is a strong predictor of satisfactory response to invasive treatment. ${ }^{8}$ According to these data, we considered severe hypertension demonstrated by ABPM an important factor for the choice of revascularization. In addition, ABPM was helpful for the demonstration of stenting efficacy during follow-up and, similarly, it could be useful for prompt detection of restenosis at long-term evaluation.

One of the least clear aspects of RAS management is, in fact, the identification of patients who are likely to benefit from percutaneous intervention: the detection of a hemodynamically significant stenosis predicts improvement in hypertension after revascularization. ${ }^{9}$ In addition to ABPM, another important clinical element of decision was the coexistence of normal glomerular filtration rate (GFR) with high circulating plasma renin (CPR) that strongly suggests that the compensatory mechanism implemented by kidney in response to RAS to keep GFR normal was fully operating in our patient and was also confirmed by the persistent normalization of GFR together with the abrupt fall of CPR after stenting, a proof of the efficacy of revascularization.

Doppler ultrasonography, in addition to being helpful for diagnosis of RAS, could be a further tool to screen patients for revascularization; a low intrarenal arterial resistance is predictive of good response to invasive treatment, since it is suggestive of a kidney without structural alterations in intraparenchymal vascularization induced by long-standing hypertension. ${ }^{10-12}$ The parvus-tardus waveform that we found upon Doppler ultrasound denotes low intrarenal arterial resistance, a parameter that normalizes immediately after stenting.

In order to make the decision of revascularization, we considered the following: recent onset hypertension, young age, severe and uncontrolled hyperten-

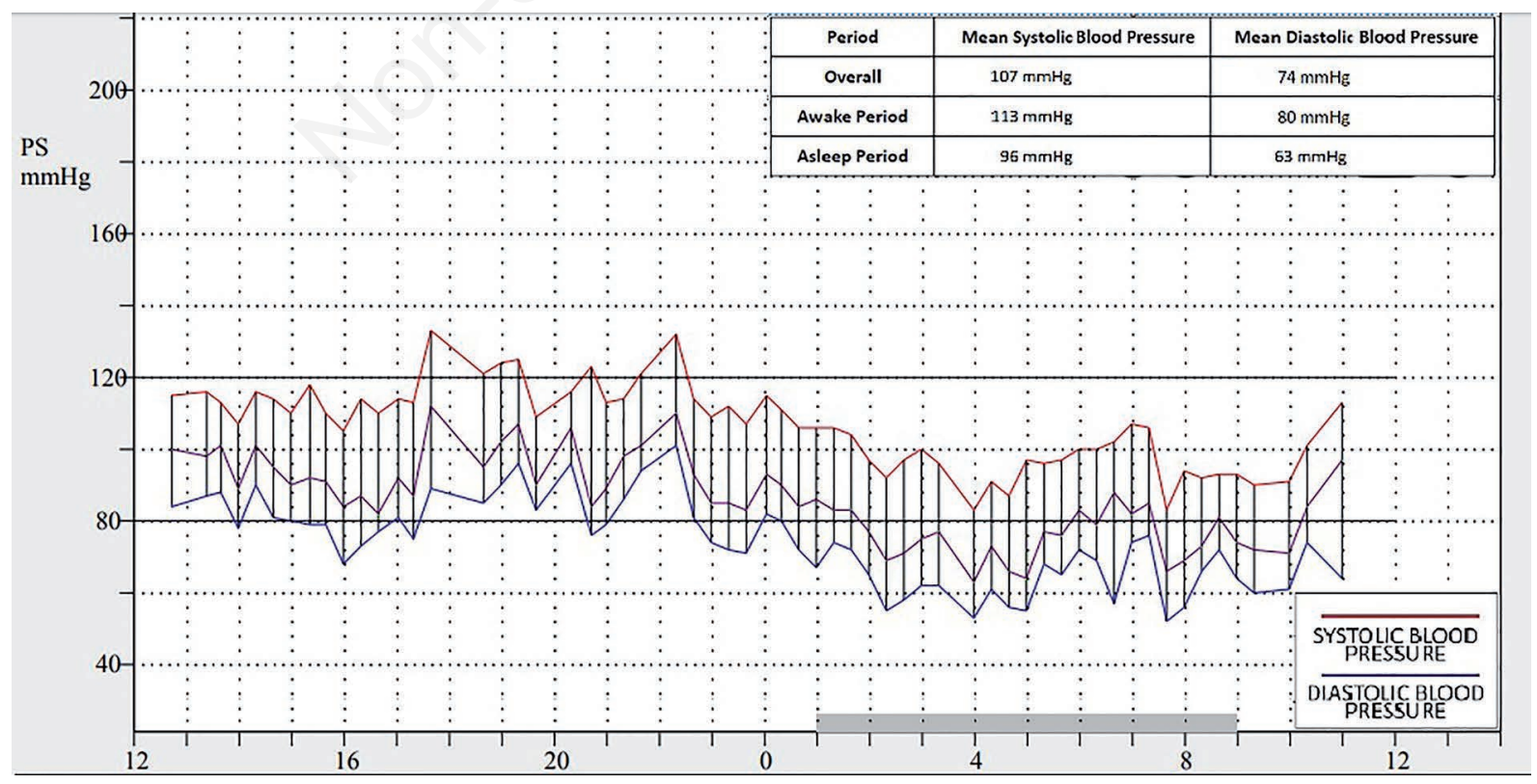

Figure 3. Ambulatory blood pressure monitoring result after 10 months of follow-up. 
sion, significant stenosis, compensatory increased activities of renin-angiotensin system.

Renal angioplasty with stent placement is a common option of treatment for renovascular hypertension, particularly in case of atherosclerotic stenosis, with low periprocedural complications and good results on blood pressure control and renal function, especially among patients with normal GFR. ${ }^{13}$

The utilization of coronary drug-eluting stent (DES) for renal artery is growing, because of the increasing and encouraging, albeit preliminary, data. ${ }^{14}$ DES might be useful in patients with small renal arteries (associated with higher risk of restenosis), and in case of in-stent restenosis, as successfully described in a case of recurrent stenosis of a bare metal stent. ${ }^{15,16}$

\section{Conclusions}

In conclusion, the diagnostic and therapeutic management of patients with renal artery stenosis is challenging. Integration of information from ABPM, intrarenal doppler evaluation, plasma renin levels and GFR is of crucial importance to characterize patients and identify the best treatment option.

\section{References}

1. Williams B, Mancia G, Spiering W, et al. 2018 ESC/ESH Guidelines for the management of arterial hypertension. The Task Force for the management of arterial hypertension of the European Society of Cardiology (ESC) and the European Society of Hypertension (ESH). Eur Heart J 2018;00:1-98.

2. Cooper CJM, Murphy TP, Cutlip DE, et al. Stenting and medical therapy for atherosclerotic renalarterystenosis. N Engl J Med 2014;370:13-22.

3. Persu A, Giavarini A, Touzé E, et al. European consensus on the diagnosis and management of fibromuscular dysplasia. J Hypertens 2014;32:1367-78.

4. de Simone G, Mancusi C, Esposito R, et al. Echocardio- graphy in arterial hypertension. High Blood Press Cardiovasc Prev 2018;25:159.

5. Funder JW, Carey RM, Mantero F, et al. The management of primary aldosteronism: case detection, diagnosis, and treatment: an Endocrine Society Clinical Practice guideline. J Clin Endocrinol Metab 2016;101:1889-916.

6. Dolan E, Stanton A, Thijs L, et al. Superiority of ambulatory over clinic blood pressure measurement in predicting mortality: the Dublin outcome study. Hypertension 2005;46:156-61.

7. O'Brien E, Parati G, Stergiou G, et al. European Society of Hypertension position paper on ambulatory blood pressure monitoring. J Hypertens 2013;31:1731-68.

8. Jujo K, Saito K, Ishida I, et al. Efficacy of 24-hour blood pressure monitoring in evaluating response to percutaneous transluminal renal angioplasty. Circ J 2016;80: 1922-30.

9. Herrmann SMS, Textor SC. Diagnostic criteria for renovascular disease: where are we now? Nephrol Dial Transplant 2012;27:2657-63.

10. Radermacher J, Chavan A, Bleck J, et al. Use of Doppler ultrasonography to predict the outcome of therapy for renal-artery stenosis. N Engl J Med 2001;344:410-7.

11. Santos SN, Leite LR, Tse TS, et al. Renal resistance index predicting outcome of renal revascularization for renovascular hypertension. Arq Bras Cardiol 2010;94:452-6.

12. Soulez G, Therasse E, Qanadli SD, et al. Prediction of clinical response after renal angioplasty: respective value of renal Doppler sonography and scintigraphy. AJR Am J Roentgenol 2003;181:1029-35.

13. Dorros G, Jaff M, Mathiak L, He T. Mulitcenter Palmaz stent renal artery stenosis revascularization registry report: four-year follow-up of 1,058 successful patients. Catheter Cardiovasc Interv 2002;55:182-8.

14. Zähringer M, Pattynama PM, Talen A, Sapoval M. Drug-eluting stents in renal artery stenosis. Eur Radiol 2008; 18:678-82.

15. Stone PA, Campbell JE, Aburahma AF, et al. Ten-year experience for in-stent stenosis. J Vasc Surg 2011;53: 1026-31.

16. Arce-Santiago M, Rodríguez-Cruz E. Treatment of a recurrent renal artery stenosis and stent fracture using a drug eluting stent in a pediatric patient. CEN Case Rep 2016;5:18-22. 